Arts

et Savoirs
Arts et Savoirs

$13 \mid 2020$

Lumières plurielles

\title{
Refaire sa bio-bibliographie
}

Le regard rétrospectif de Mme de Genlis sur ses ouvrages éducatifs dans ses Mémoires

How to write a bio-bibliography: the retrospective look of Mme de Genlis on her educational books in her Mémoires

Marie-Emmanuelle Plagnol-Diéval

\section{OpenEdition}

Journals

Édition électronique

URL : http://journals.openedition.org/aes/2532

DOI : 10.4000/aes.2532

ISSN : 2258-093X

Éditeur

Laboratoire LISAA

Référence électronique

Marie-Emmanuelle Plagnol-Diéval, «Refaire sa bio-bibliographie », Arts et Savoirs [En ligne], 13 | 2020,

mis en ligne le 01 juin 2020, consulté le 19 juin 2020. URL : http://journals.openedition.org/aes/2532 ; DOI : https://doi.org/10.4000/aes.2532

Ce document a été généré automatiquement le 19 juin 2020.

Centre de recherche LISAA (Littératures SAvoirs et Arts) 


\section{Refaire sa bio-bibliographie}

Le regard rétrospectif de Mme de Genlis sur ses ouvrages éducatifs dans ses Mémoires

How to write a bio-bibliography: the retrospective look of Mme de Genlis on her educational books in her Mémoires

Marie-Emmanuelle Plagnol-Diéval

1 Dans le cadre d'une réflexion générale sur " les Lumières au pluriel », les Mémoires ${ }^{1}$ de Mme de Genlis, offrent un angle intéressant, non comme source autobiographique ou historique même si le texte s'y prête aisément, mais comme illustration de l'écart entre le contenu des œuvres éducatives qui jalonnent la carrière de l'auteure et le reflet qu'en donnent les volumes de 1825. La position de Mme de Genlis par rapport aux notions de Lumières chrétiennes, catholiques, à la mouvance des antiphilosophes, voire des anti-Lumières, reste ambiguë. Dans le cadre des recherches sur les femmes éducatrices et de leur rapport à la religion et à la raison, nous nous étions déjà intéressé au texte composé pour la première communion de Louis-Philippe, La Religion considérée comme l'unique base du bonheur et de la véritable philosophie, l'ouvrage religieux, apologétique, programmatique, publié en $1787^{2}$, qui ouvre les hostilités avec les philosophes des Lumières.

2 Notre hypothèse aujourd'hui est que le rapport à la religion et à la politique dans la perspective pédagogique évolue au fil des décennies et comment Mme de Genlis tente de bâtir a posteriori une position cohérente quand elle évoque ses ouvrages éducatifs. En effet, autant ses préfaces renseignent sur ce qu'elle veut dire et défendre au moment de la publication du livre ou peu de temps après, dans une temporalité d'attaquesréponses assez brève dont elle s'est fait une spécialité, autant les mémoires portent un regard à distance, de plusieurs années, voire décennies sur une œuvre éducative qui s'étend sur toute la carrière, de surcroît dans des contextes politiques et idéologiques fluctuants. Analyser comment les Mémoires réécrivent la présentation, le contenu et la réception des ouvrages d'éducation, parus pour les premiers environ 40 ans auparavant, décrypter les interprétations mises en place postérieurement permettent de voir quelles fonctions complexes l'ancien "gouverneur » entend donner à ses 
souvenirs. Si leur titre insiste sur la valeur testimoniale, «depuis 1756 jusqu'à nos jours », soit la traversée d'une profusion de régimes politiques et de changements divers, nul n'oubliera que l'auteure a vécu de sa plume, certes aidée par des pensions de diverses origines, mais aussi qu'elle a joué un rôle politique non négligeable, souvent indirect, toutefois émaillé d'actions fortement symboliques.

C'est pourquoi la publication en 1825 de ce massif mémoriel a pour fonction essentielle de tirer un fil d'Ariane à travers le maquis des circonstances ayant présidé aux œuvres, de retrouver une unité plausible, sinon factice aux textes éducatifs, dont les préceptes invoqués et les exemples mis en scène ont partie liée avec des options philosophiques, morales, religieuses, sociales et politiques.

\section{Repérage significatif des notations sur les ouvrages éducatifs}

4 Si les Mémoires ne sont pas très bavards sur les ouvrages pédagogiques, le relevé des occurrences donne néanmoins un aperçu à peu près complet, y compris des ouvrages restés manuscrits ou disparus, comme ces Réflexions d'une mère de vingt ans, sorte d'avant-texte d'Adèle et Théodore, qui souligne les étapes que Mme de Genlis entend donner à sa vie et à sa carrière, ainsi que le passage du biographique au romanesque ${ }^{3}$.

J'avais commencé depuis quelques mois un ouvrage que j'intitulai Réflexions d'une mère de vingt ans, quoique je n'en eusse que dix-neuf. Cet ouvrage, que j'ai perdu vingt-cinq ans après avec tant d'autres manuscrits, n'avait rien de romanesque, et j'en ai pris par la suite beaucoup d'idées que j'ai mises dans Adèle et Théodore. ${ }^{4}$

Sont ainsi évoqués ou analysés le Théâtre d'éducation, Les Veillées du château, Adèle et Théodore, les Leçons d'une gouvernante (à travers de très nombreuses références), Le Petit La Bruyère ${ }^{5}$, l'Herbier moral ${ }^{6}$ également composé durant l'émigration, cette fois à Brevel, les Heures à l'usage des jeunes personnes ${ }^{7}$, la nouvelle Ida ou le jupon vert ${ }^{8}$. Ces évocations sont inégalement réparties selon les tomes: peu nombreuses dans le tome I (qui concerne la période avant l'entrée en écriture) elles se concentrent dans les tomes II et III qui correspondent à une intense période d'écriture, puis diminuent dans les tomes IV et $\mathrm{V}$ principalement tournés vers la Révolution et l'émigration.

6 Le deuxième constat est que pour les ouvrages ayant bénéficié d'une bonne réception, dénuée de polémiques - fait rare dans son œuvre - le filtre le plus utilisé est celui du lien autobiographique. Deux explications semblent pouvoir être avancées. Mme de Genlis écrit des mémoires et donc se coule dans les impératifs du genre qui bénéficie d'une forte attente de la part du public et d'une certaine codification éditoriale. Elle n'entend pas se présenter comme une femme de lettres, mais comme une éducatrice que la vie et les expériences pédagogiques ont amenée à produire une œuvre. C'est ainsi que dans le tome I, les pièces du Théâtre à l'usage des jeunes personnes sont simplement évoquées, à l'occasion des souvenirs les ayant directement inspirées, sans que leur fond soit analysé. Mme de Genlis s'en tient au plan anecdotique concernant ses premiers souvenirs parisiens transposés ${ }^{9}$ :

Tous ces supplices [entre autres deux dents arrachées, des souliers étroits, des papillotes, un panier, un collier de fer, des besicles et un maître de maintien] me firent une telle impression, que je ne les ai jamais oubliés, et que je les ai fidèlement dépeints depuis dans la petite comédie de mon Théâtre d'éducation, intitulée La Colombe. ${ }^{10}$ 
7 C'est également le cas de La Rosière de Salency qui prend place dans le tome IV de 1780 consacré aux « enfants de marchands et d'artisan $»^{11}$ pour y illustrer la vertu paysanne sacralisée par l'institution civile et religieuse. Les Mémoires notent simplement le poème de M. de Sauvigny, puis la « comédie » de Mme de Genlis et le procès « 7 ou 8 ans après » des « rosières avec leur seigneur » (procès dans lequel Mme de Genlis a joué un rôle actif $\left.{ }^{12}\right)$.

8 Au-delà de ces anecdotes, le lecteur apprend au fil des souvenirs la genèse de certains textes comme " l'épisode de Cécile » inséré dans Adèle et Théodore, né de la rencontre au couvent d'Origny de Mme de Rochefort devenue religieuse contre son gré, apparemment consolée de son sort, mais qui tombe dans une " consomption mortelle » à la vue du bonheur familial et champêtre d'une paysanne nommée Nicole ${ }^{13}$. Certaines évocations renseignent sur les modalités de composition. C'est le cas du passage des Mémoires relatifs à un morceau inséré dans Les Annales de la vertu. Ce témoignage figure à la suite de l'abrégé géographique de l'Amérique et concerne les mœurs des « sauvages de la Louisiane ${ }^{14}$. Intitulé « Extrait d'un manuscrit », il est précédé d'une dizaine de lignes justificatives et éclairé par une note ${ }^{15}$ dans laquelle Mme de Genlis relate la vie de M. de Mézières, «qui, envoyé aux îles à treize ans, s'échappa, alla vivre parmi les sauvages » et noua des relations avec les Espagnols. Mme de Genlis eut connaissance du manuscrit lors d'un voyage de son auteur à Paris à 1774 et n'y a « fait aucune espèce de changement $»^{16}$. Dans les Mémoires comme dans la note des Annales, elle regrette d'avoir dû en retrancher la moitié : «Ce morceau, quand cet ouvrage parut, fut très remarqué ; on regretta qu'il n'eût pas plus d'étendue; je n'y avais pas changé un seul mot. ${ }^{17}$ Enfin, les Mémoires attestent la véracité du «trait », souvent explicitée par une note de Mme de Genlis en bas de page et indiquent comment l'anecdote a ensuite pris place dans un texte éducatif. Le texte mémoriel, une nouvelle fois, renseigne sur la fabrique d'écriture. Parmi les nombreux exemples, citons dans l'ordre chronologique des souvenirs évoqués dans les Mémoires :

- les actions charitables de Mlle de Raffeteau auprès d'une pauvre paralytique ${ }^{18}$ qui prennent place dans Les Veillées du château (1782)

- la rencontre des religieux lors du voyage à Naples ${ }^{19}$ qui inspire la nouvelle «Les Hermites des Marais Pontins $»^{20}$,

- la rencontre à Forges de la famille pauvre et vertueuse ${ }^{21}$ à l'origine de la nouvelle «Les Solitaires de Normandie ", insérées dans Les Veillées du château ${ }^{22}$,

- les retrouvailles à Spa de Jeannette ${ }^{23}$, l'héroïne de la pièce de théâtre, L'Aveugle de Spa ${ }^{24}$.

\section{Typologie des références : du souvenir à la revendication}

9 Si les souvenirs semblent se succéder selon les caprices de la mémoire et la simple remise en ordre chronologique ou thématique, il n'en est rien et une stratégie précise guide les évocations des œuvres, leur ordre et leur teneur. Rappeler les circonstances de composition des ouvrages permet de se les réapproprier, surtout si leur succès leur a fait connaître d'autres formes d'exploitation dans lesquelles le nom de Mme de Genlis a plus ou moins disparu. En deux pages, Mme de Genlis rappelle qu'elle est l'autrice de la nouvelle Les Solitaires de Normandie, qui a donné lieu à un " vaudeville à succès " ${ }^{25}$, en un acte d'Augustin de Piis et Pierre-Yvon Barré, créé le 4 mai $1790^{26}$ et inspiré des vers à Antoine-Marin Le Mierre ${ }^{27}$. 
Outre ce traçage des œuvres, le souvenir consigné permet de s'interroger sur l'acquisition progressive du statut d'auteure. Le tome III revient avec précision sur les circonstances de la publication des pièces du Théâtre d'éducation ${ }^{28}$. Mme de Genlis s'y résout en 1779 pour aider les trois frères nobles répondant au patronyme de Queissat ${ }^{29}$. Les Mémoires insistent sur l'autorisation de $\mathrm{M}$. de Genlis et le succès (vente rapide des exemplaires, sommes importantes données par les acheteurs, négociations financières et libération des frères). Une note ${ }^{30}$ ambiguë précise comment s'effectue cette entrée en littérature mi-charitable, mi-mondaine, mi-éducative :

Il est vrai qu'il y eut un assez grand nombre d'exemplaires en papier vélin et qu'il y avait à ce volume une fort jolie vignette, parfaitement bien gravée, représentant ma devise, qui se rapportait à mes enfants, parce que je n'écrivais pour leur éducation que durant la nuit depuis minuit jusqu'à trois ou quatre heures du matin. Cette devise offrait pour corps une lampe posée sur un bureau à côté d'une écritoire, elle avait pour âme ces mots Pour éclairer je me consume.

Les pages suivantes soulignent la réception de l'ouvrage, les traductions, les éloges divers ( Quand je publiai mon premier volume du Théâtre d'éducation, ce volume libérateur de MM. de Queissat, il y eut pour moi un enthousiasme général et dans la société, et parmi les littérateurs $»^{31}$ et énumèrent les réactions des journalistes, les innombrables visites "entre autres de $\mathrm{M}$. de La Harpe $»^{32}$, l'édition bilingue de l'impératrice de Russie ${ }^{33}$, les témoignages amicaux de l'électrice de Saxe, de « la reine et toutes les princesses " à Versailles qui semblent donner raison à l'affirmation "Enfin, jamais on n'est entré dans la carrière des lettres avec plus d'éclat et de bonheur $»^{34}$. Toutefois, on remarque que Mme de Genlis mêle à ces considérations sur sa notoriété la chronologie de la naissance des jumelles d'Orléans ${ }^{35}$, les visites quotidiennes qu'elle leur rend, sa vocation pédagogique et sa décision de quitter le monde (à 31 ans, en $1777^{36}$. La suite des pages annonce de manière simultanée l'installation à Bellechasse, les aménagements intérieurs dont le fameux décor pédagogique ( j’ai détaillé toutes ces choses dans Adèle et Théodore $\|^{37}$ ), et la publication dans les dix premiers mois de Bellechasse des autres volumes du Théâtre d'éducation, assortie en note des éloges de Grimm $^{38}$.

12 C'est dire que la posture est complexe et savamment préservée dans le texte mémoriel : celle d'une femme éducatrice, auteure reconnue, immédiatement prête à renoncer, au moins en apparence, aux suffrages pour se consacrer à sa tâche d'éducatrice et bientôt de "gouverneur", autre forme de reconnaissance et de consécration genrée et professionnelle.

Les Mémoires sont en effet le lieu de la justification pédagogique a posteriori, même si celle-ci a été conduite de manière quasi systématique au fur et à mesure des œuvres publiées. Ainsi à propos de la vague de journaux présidant à l'éducation des enfants d'Orléans, Mme de Genlis détaille son système ${ }^{39}$, qualifié de " panoptique » par Philippe Lejeune $e^{40}$, tant il instaure une surveillance globale et démultipliée. Les Mémoires rappellent en effet que l'éducation des enfants d'Orléans est consignée par Charles Gardeur-Lebrun dans un journal qui donne un compte rendu détaillé de tout ce qui se passe quand il est avec les princes, que ce journal est ensuite annoté en marge par Mme de Genlis qui ne communique avec le sous-gouverneur que par ce biais ${ }^{41}$. De son côté, elle-même tient un journal dont chaque article est signé par les princes et par Adélaïde $^{42}$ : ce journal est conçu pour le duc et la duchesse, mais comme, selon Mme de Genlis, ils «n'ont jamais voulu [le] lire » ${ }^{43}$, elle l'édite " étant encore en France en $1790 »^{44}$, «sous les yeux du duc et de la duchesse $»^{45}$ dans les Leçons d'une gouvernante, 
ouvrage de justification éducative, familiale et politique. Les notes de bas de page ${ }^{46}$ sur la circulation de ces journaux montrent dans quel climat politique et personnel ils s'insèrent: Mme de Genlis les confie en 1791 à un notaire autorisé à les faire lire à qui veut pour vérifier leur conformité avec les Leçons d'une gouvernante, qui les lui rend à son retour d'émigration. Elle les remet ensuite, toujours manuscrits, au duc d'Orléans ${ }^{47}$.

Non contents de rétablir la chronologie des événements dans un contexte politique si chargé, les Mémoires ont pour fonction, après les Leçons d'une gouvernante, d'absoudre Mme de Genlis des querelles avec les sous-gouverneurs et avec la duchesse d'Orléans comme de prolonger la réflexion entamée par les textes éducatifs. Par exemple, le passage relatif aux journaux éducatifs de Bellechasse et des autres lieux de résidence se prolonge par une vingtaine de pages ${ }^{48}$ qui donnent les sujets de "compositions" proposés presque quotidiennement aux élèves, assortis de la partie rédigée par l'institutrice elle-même ${ }^{49}$. De même, Mme de Genlis publie un extrait de la lettre qu'elle adresse à Mlle de Chartres au moment où elle la quitte en émigration : cette lettre comporte, entre autres, une liste de livres, qui doit être lue en regard avec le plan de lecture d'Adèle dans Adèle et Théodore ${ }^{50}$. Les Mémoires offrent donc un espace de publication à ce qui était réservé au privé éducatif, ils offrent un supplément aux textes publiés relatifs à l'éducation dispensée par Mme de Genlis aux enfants d'Orléans. On doit bien sûr s'interroger sur la publicité donnée à ces textes insérés en 1825 . Concernant les extraits des journaux et l'examen de conscience, un premier élément de réflexion se trouve sans doute dans le contenu qui commence comme un manuel de conduite, adapté à la situation sociale des élèves et s'achève par un questionnaire, rédigé par Mme de Genlis pour ses élèves, dans la pure tradition catholique. Une note en précise l'utilisation ( « je donnai cet examen de conscience à M. Lebrun, qui, depuis ce temps-là, l'a lu tous les soirs à nos élèves $\|^{51}$ ) et le rattache chronologiquement et thématiquement à la composition de La Religion considérée comme l'unique base du bonheur et de la véritable philosophie. Ils constituent en 1825 un réseau de textes et de réflexions qui promeuvent l'image d'une éducation catholique et apolitique dispensée aux enfants d'Orléans. Une seconde explication est liée aux rapports entre Mme de Genlis et le duc de Chartres devenu duc d'Orléans. Vis-à-vis de l'élève préféré, mis en avant durant la Révolution, au centre d'un certain nombre de textes de reniement politique comme la lettre de Silk, il importe en ces années 1820 de restaurer le lien avec la gouvernante. Ainsi s'explique peut-être la relation de ces «traits» comme celui-ci, intitulé "Désintéressement de M. le Duc de Chartres », et le témoignage de l'intérêt affectif et pédagogique de Mme de Genlis vis-à-vis d'Adélaïde, au moment de la séparation.

Quoi qu'il en soit, le rapport entre les œuvres éducatives et les Mémoires est fortement affirmé, comme l'affirme Mme de Genlis à propos des innovations pédagogiques énoncées dans les Leçons d'une gouvernante " qu'il est nécessaire de lire, avant ou après ces mémoires, si l'on veut avoir une idée exacte de l'éducation que j'ai donnée à ces princes $»^{52}$.

\section{Mise en avant d'une continuité religieuse, politique, morale, sociale et pédagogique}

Les positions religieuses de Mme de Genlis, qui font l'objet de tant de discussions et qui ont été si diversement commentées par les contemporains et par la critique, sont bien sûr au centre des remises en perspective effectuées par les Mémoires. Ceux-ci, en 
s'efforçant de ne pas opérer de coupes trop visibles dans les événements et les ouvrages jalonnant la carrière et la vie, plaquent a posteriori une continuité, notamment celle de la fidélité religieuse qui est sans doute assez juste si l'on regarde les textes, mais qui ne s'exprime pas avec la même vigueur ${ }^{53}$ (comme le montre un parallèle entre le théâtre et La Religion... pourtant chronologiquement proches). Les Mémoires, publiés en pleine Restauration, postulent cette continuité religieuse et la prépare dès l'entrée en écriture. Ainsi, dès le tome III, la démonstration est amorcée avec la visite de D’Alembert («pour lequel je n'avais aucun pendant naturel $»^{54}$ ) en compagnie de M. de Schomberg, qui, après le tome des Pièces tirées de l'Écriture Sainte, lui conseille " amicalement» de renoncer à parler de religion (une "mode passée») et lui fait miroiter le projet de placer quatre femmes à l'Académie-Française, elle, Mmes de Montesson, d'Angevillers et d'Houdetot ${ }^{55}$. Mme de Genlis rapporte longuement sa réponse d'alors et la discussion très vive qui s'ensuit, sa propre fermeté :

Je répondis qu'il m'était impossible de séparer la religion de la morale, et que je n'aurais aucune espèce de talent, si je voulais la priver d'une telle base ; que non seulement je parlerais sans cesse de la religion, mais que je combattrais de tous mes faibles moyens la fausse philosophie qui l'attaque et la calomnie. Il me répondit avec colère et avec dédain, que je m'en repentirais. Il ajouta du ton le plus ironique et le plus amer que la grâce pourrait être de mon côté mais que la force n'y serait pas. Je répondis qu'avec la raison, la droiture et la persévérance, on est toujours fort. La dispute devint très piquante de part et d'autre, malgré tous les efforts de $\mathrm{M}$. de Schomberg pour nous adoucir et nous concilier. D'Alembert s'en alla furieux contre moi ; depuis ce jour-là je ne l'ai plus revu. Tel a été le commencement de ma brouillerie avec les philosophes. ${ }^{56}$

Les publications suivantes, selon Mme de Genlis, creusent le fossé entre ses admirateurs et les philosophes. Adèle et Théodore recueille le «suffrage du public» et s'attire la " haine irréconciliable de tous les prétendus philosophes et de leurs partisans ${ }^{57}$. Or, il est intéressant de constater qu'elle grossit le trait anti-philosophique, fausse en partie le contenu et la réception contemporaine ${ }^{58}$. Elle surinterprète ainsi les lettres de $\mathrm{M}$. Lagaraye à Porphire, deux personnages du roman, «dans lesquelles [écrit-elle] j'ai prédit et dépeint tout ce que j'ai éprouvé depuis $"^{59}$ et peut ainsi établir un continuum depuis le refus fait à D'Alembert et celui réitéré à l'abbé Cerutti de continuer à donner, sous le nom de "Lettres de Marie-Anne", au début de la Révolution, des pages de morale sans religion à La Feuille villageoise ${ }^{60}$. L'affirmation de sa position et sa constance permettent de dénoncer les complots et des injustices dont elle se dit la victime, qu'il s'agisse de la critique d'Adèle et Théodore parue dans Le Mercure ${ }^{61}$ que La Harpe confie à l'abbé Joseph-Honoré Rémi et qui aurait été en réalité écrite par D’Alembert ou du prix Montyon attribué à Mme d'Épinay. Selon elle, l'ouvrage récompensé n'aurait pas dû l'être puisqu'il n'est pas nouveau (il s'agit pour elle d'une seconde partie...) et l'ouvrage écarté serait ses Veillées du château (et non Adèle et Théodore) à cause des deux nouvelles, Le Palais de la vérité (qui attaque la philosophie) et Les Deux réputations (qui critique «les contes prétendus moraux de Marmontel $\aleph^{62}$ ). Mais surtout la décision s'explique par la divergence religieuse des deux éducatrices : « Mais madame d'Épinay était philosophe, et elle s'est bien gardée de parler de religion à son Émilie " ${ }^{63}$. De manière générale, les Mémoires se veulent le lieu de la proclamation morale, de l'explication synthétique et du dévoilement de la vérité cachée ou mise à mal jusque-là.

D'un point de vue moral, Mme de Genlis, comme tant d'autres, utilise les Mémoires comme espace de réhabilitation. C'est pourquoi tant de pages sont consacrées aux Leçons d'une gouvernante, texte qui tente d'expliquer sa conduite envers la duchesse 
d'Orléans. La répartition matérielle (quantitative) de sa défense entre les Leçons d'une gouvernante, le Journal d'éducation et les Mémoires est certes complexe, mais la présentation qu'elle en fait est faussée. Un passage éclaire particulièrement sa stratégie. Les éléments de ce véritable feuilleton de rupture, de divorce familial et éducatif se décomposent en trois temps. Apparemment, tout a été dit dans les Leçons :

J'ai donné, dans les Leçons d'une gouvernante, le détail le plus circonstancié de ma conduite dans cette triste conjoncture. C'est là que l'on pourra voir toute la pureté de mes intentions, et les efforts inouïs que j'ai faits pour lui [la duchesse] gagner et lui conserver le cœur de ses enfants, malgré toutes les injustices que j'éprouvais.

Mme de Genlis précise qu'elle a tu les faits dans le fameux Journal d'éducation « afin que mes élèves n'en eussent point connaissance et qu'ils ne pussent altérer en rien les sentiments que je leur désirais pour leur vertueuse mère ». Cependant, elle entend "tracer ici [dans les Mémoires] un rapide abrégé ${ }^{64}$. Or que font les Mémoires? Ils recopient fidèlement les lettres échangées entre les trois protagonistes (une lettre de Mme de Genlis à la duchesse ${ }^{65}$ ), relatent les démarches du duc envers la duchesse concernant le partage du temps passé avec les enfants entre les deux femmes, sorte de de garde alternée avant l'heure ${ }^{66}$, reproduisent une lettre de Mme de Genlis directement à la duchesse sans passer par le duc sur l'héritage moral qu'elle lui laisse après l'éducation du fils aîné ${ }^{67}$.

L'historique des options politiques diffusées dans les textes éducatifs se caractérise par un équilibre tout aussi difficile. Mme de Genlis ne peut passer sous silence les Discours des années 1790, que l'on peut caractériser de très réformateurs et qui bien sûr ne peuvent se légitimer en pleine Restauration. Que faire de ces textes embarrassants dans sa bio-bibliographie reconstruite ? La première chose est de minorer leur importance :

Je donnai successivement mes Discours moraux sur l'éducation de monseigneur le dauphin; sur les écoles cloîtrées, que je proposais pour remplacer les couvents de religieuses, dont je déplorais la perte; sur l'éducation du peuple; sur la gymnastique appliquée à l'éducation; sur l'éducation publique, etc., etc. Tous ces discours parurent dans le cours de l'année 1790 ; ils sont réunis en un volume et sont réunis dans mes œuvres. ${ }^{68}$

La seconde est de les vider de leur contenu ou de l'infléchir : ainsi le commentaire sur le Discours sur l'éducation du dauphin passe-t-il sous silence la proposition de l'adoption, de même que le commentaire sur celui des couvents adoucit la proposition de suppression.À ses ennemis qui l'accusent de divergences politiques, Mme de Genlis oppose une réponse identique de soi-disant constance morale et politique: elle a toujours été contre les abus et a toujours été royaliste :

Dans tous les temps, j'ai toujours eu des principes monarchiques, et j'ai été attachée à la race royale, comme le prouvent tous mes ouvrages. Dans l'émigration, j'ai montré ces sentiments dans Les Chevaliers du Cygne, dans Les Petits émigrés; enfin sous l'empire de Napoléon, j'ai remis Louis XIV à la mode dans La Duchesse de La Vallière et Madame de Maintenon. ${ }^{69}$

Cette pseudo-stabilité et ce pseudo-effacement de la politique au profit de la morale lui permettent d'avancer qu'elle a diversement réagi face aux événements révolutionnaires dès la prise de la Bastille : une assertion invérifiable, que pourraient infirmer les faits et qui se fonde sur une apophétie facile :

Je n'étais d'aucun parti, que de celui de la religion ; je désirais la réforme de certains abus, et j'ai vu avec joie la démolition de la Bastille, l'abolition des lettres de cachet et des droits de chasse : c'était tout ce que j'avais désiré, ma politique n'allait pas au-delà de cela. En même temps, personne plus que moi n'a vu avec douleur et 
horreur les excès qui ont été commis, dès les premiers moments de la prise de la Bastille, dont, comme je l'ai dit, je n'ai aimé que la démolition. ${ }^{70}$ du futur Louis-Philippe :

$1^{\circ}$. Que je ne me suis jamais mêlée d'affaires. $2^{\circ}$. qu'il ne m'a jamais parlé des siennes que vaguement. $3^{\circ}$. que, depuis la révolution, il a totalement cessé de m'en parler $; 4^{\circ}$. que je ne connais aucun de ses gens d'affaires, pas même de vue $; 5^{\circ}$. que je n'ai eu connaissance de ses Cahiers imprimés que lorsqu'ils ont été publiés. ${ }^{71}$

Après le politique, la troisième forme de continuité est morale, sociale et pédagogique. Apolitique ou presque, uniquement guidée par la religion, la morale et sa passion éducative, Mme de Genlis peut se carrer dans ce personnage idéal et cohérent de gouverneur qu'elle ne cesse de vouloir incarner. Elle réaffirme par conséquent que ses ouvrages sont en accord avec ses principes, qu'elle a dispensé gratuitement l'éducation des trois princes ${ }^{72}$. Elle proclame son désintéressement en citant les dernières pages de son Précis de conduite $e^{73}$ et rappelle qu'elle a toujours refusé les honneurs, hormis cette "députation [...] des six corps des marchands de Paris avec une lettre signée d'eux » pour le « volume du Théâtre d'éducation pour les enfants du peuple »" ${ }^{74}$. Elle réaffirme sa foi en l'amitié et la reconnaissance, sa déception devant la prétendue réaction misogyne du chevalier de Bonnard à la parution d'Adèle et Théodore ${ }^{75}$, cet ouvrage, qu'elle conçoit apparemment comme le prototype de sa mission en soulignant combien il a fait pour la critique des mœurs, la «mode honteuse » du parfilage ${ }^{76}$ et dont elle se loue en ces termes: «il y a eu peu d'ouvrages dont les critiques aient eu plus d'influence sur la société $»^{77}$.

L'éducation singulière que reçut Mme de Genlis de la part de ses parents et de Mlle de Mars, son institutrice, est détaillée avec une certaine complaisance dans le premier tome des Mémoires ${ }^{78}$. Sans aucun doute, la future éducatrice et auteure y puisa sa volonté d'autodidacte, sa passion de l'enseignement, illustrée par la scène emblématique avec les petits garçons du voisinage ${ }^{79}$, développée ensuite avec ses enfants et ses différents élèves, idéalisée et en partie théorisée dans ses ouvrages. Sa proximité avec les sphères de pouvoir, le rang de ses élèves et les événements expliquent que ses Mémoires, rédigés en pleine Restauration, aient pour enjeu la reconstruction idéale d'une logique de vie, d'actions et d'idées, y compris et surtout quand elle évoque ses ouvrages éducatifs. Certes, les péritextes ont commencé ce travail, certes les échanges conflictuels avec les critiques et la presse des contemporains ont renforcé ces prises de position, mais seuls les volumes de 1825 peuvent mettre en œuvre cette reconstruction globale d'une continuité religieuse et morale, politique et sociale en s'appuyant sur le lien entre la biographie et l'œuvre, le parcours des œuvres ou la pratique de l'auto-extrait.

\section{NOTES}

1. Nous nous limiterons aux cinq premiers volumes des Mémoires inédits de madame la comtesse de Genlis sur le dix-huitième siècle et la Révolution française depuis 1756 jusqu'à nos jours, Paris, 
Ladvocat, 1825, 10 vol. et plus précisément à la date du retour en France après l'émigration ( «Je retournai à Paris ", tome V, p. 84, désormais, V, 84). C'est en effet dans ces cinq volumes que se trouvent les références les plus pertinentes aux ouvrages éducatifs qui occupent la majeure partie des premières productions genlisiennes. Nous entendons par ouvrages éducatifs ceux qui sont explicitement destinés à un lectorat d'enfants, de jeunes et d'adultes en position d'enseignant (parents ou professionnels).

2. Notre édition de référence est La Religion... in CEuvres complètes de Mme la comtesse de Genlis, Maestricht, J.-P. Roux et Cie, 1787, tome XVIII. Voir Marie-Emmanuelle PlagnolDiéval, « Mme de Genlis ou le basculement : La Religion considérée comme l'unique base du bonheur et de la véritable philosophie (1787) », section » Les Lumières chrétiennes à la frontière entre deux camps? Pour une conception des Lumières au pluriel ", 10. Kongresses des Frankoromanistenverbands 28. September bis 1. Oktober 2016 Universität des Saarlandes, Saarbrücken, Grenzbeziehungen - Beziehungsgrenzen (Liaisons frontalières), à paraître.

3. Sur l'appartenance générique d'Adèle et Théodore, Mme de Genlis émet diverses opinions, notamment celle que ce texte n'est ni un roman à clés, ni même un roman (Mémoires, III, 80).

4. Ibid., I, 238.

5. Mme de Genlis écrit en émigration, à Tournay, des pensées détachées qui sont placées ensuite dans cet ouvrage, Ibid., IV, 185.

6. Ibid., IV, 357.

7. Composées en émigration, Ibid., V, 14.

8. Ibid., V, 38-39.

9. Ibid., I, 12-13.

10. Ibid., I, 13. La Colombe figure au tome II de l'édition du Théâtre..., Paris, Chez les libraires associés, 1780 .

11. Théâtre, Paris, Chez les libraires associés, 1780, Préface, p. 3.

12. Elle écrit un mémoire pour le prieur de Salency lorsque le seigneur Danré veut empiéter sur les droits des Salenciens en nommant seul la rosière. Les habitants sont défendus par Maître Target dans un Plaidoyer en faveur de la rosière, pour les syndic et habitants du village de Salency, contre le Sieur Danré, seigneur dudit Salency, Paris, impr. de Knapen, 1774. Voir Mémoires, I, 250-251 et l'article "Fête de la Rosière », Marie-Emmanuelle Plagnol, Dictionnaire des femmes des Lumières, Huguette Krief et Valérie André (dir.), Paris, Honoré Champion, 2015, tome I, p. 479-483.

13. Mémoires, I, p. 175-176.

14. Il figure au tome IV des Annales, dans notre édition de référence, Lecointe et Durey, 1825, p. 42-51.

15. Ibid., p. 42.

16. Ibid., p. 43.

17. Mémoires, I, 118. «Mademoiselle de Raffeteau la peignait, lui lavait les pieds et lui coupait les ongles; lorsque la gouvernante n'était pas contente de son élève, elle la privait du bonheur d'exercer ces pieux devoirs de charité, et les remplissait elle-même ; cette pénitence fut la seule que mademoiselle de Raffeteau reçut, et qui lui causait la plus vive affliction. »

18. Ibid., I, 359.

19. Ibid., III, 50-51.

20. Nouveaux contes moraux et nouvelles historiques, Paris, Maradan, 1802, 3 vol. , tome II.

21. Mémoires, III, 71.

22. Sur ces nouvelles et leurs modifications au fil des rééditions, voir MarieEmmanuelle Plagnol-Diéval, «De l'anecdote vertueuse à la nouvelle édifiante: naissance d'un genre au Tournant des Lumières ", La Nouvelle de langue française aux 
frontières des autres genres, $d u$ Moyen-Âge à nos jours, volume premier, actes du colloque de Metz, juin 1996, Vincent Engel et Michel Guissard (dir.), Quorum, Presses de Belgique, 1997, p. 183-196.

23. Mémoires, III, 206.

24. Théâtre..., tome II, édition de 1780.

25. Mémoires..., III, 72.

26. La Suite des solitaires de Normandie, divertissement en un acte en vaudeville, par M. Piis..., représenté pour la première fois à Paris sur le théâtre des Comédiens Italiens... le 4 mai 1790, Paris, Vente, 1790. Le site CESAR indique quatre représentations en 1790.

27. Mémoires..., III, 72, note 1 .

28. Ibid., III, 79.

29. L'argent tiré de la publication aide les frères Queissat à payer une amende à un négociant que l'un d'entre eux avait blessé après une altercation, Mémoires, tome III, p. 73-80.

30. Ibid., III, 80, note 1 .

31. Ibid., III, 84-85.

32. Ibid., III, 85.

33. Ibid., III, 85.

34. Ibid., III, 86 .

35. Ibid., III, 80.

36. Ibid., III, 86.

37. Ibid., III, 102.

38. Ibid., III, 103, note 1.

39. Ibid., III, 149-150.

40. «Le panoptique de Madame de Genlis », Le Temps des femmes. Textes mémoriels des Lumières, Anne Coudreuse, Catriona Seth (dir.), Paris, Classiques Garnier, 2014, p. 45-68.

41. Sur l'interaction entre le gouverneur et le sous-gouverneur, nous renvoyons à l'édition Journaux de voyage et d'éducation: Spa, été 1787 / Louis-Philippe d'Orléans et Charles Gardeur-Lebrun ; édition critique par Isabelle Havelange, préface de Dominique Julia, Paris, Classiques Garnier, 2015.

42. Mémoires, III, 271.

43. Ibid., III, 271.

44. Ibid., III, 150.

45. Ibid., III, 272.

46. Ibid., III, 272.

47. Ibid., III, 150.

48. Ibid., III, 272-291.

49. «Comment on doit se conduire avec ses amis » (III, 272-278), «De la sûreté dans la société » (III, 279-282) et «Instruction sur ce qu'ils doivent aux personnes employées à leur éducation » (III, 282-286). Cette dernière partie justifie la conduite de Mme de Genlis vis-à-vis de tout le personnel éducatif des enfants d'Orléans ainsi que son propre désintéressement...

50. Ibid., IV, 267-268.

51. Ibid., III, 287, note 1.

52. Ibid., III, 160.

53. Voir Marie-Emmanuelle Plagnol-Diéval, «Le théâtre de madame de Genlis, une morale chrétienne sécularisée ", Dix-Huitième siècle, «Le Matérialisme des Lumières ", 1992, n² 24, p. 367-382.

54. Mémoires, III, 102. 
55. Ibid., 103-104.

56. Ibid., 103-104.

57. Ibid., 145.

58. Voir Marie-Emmanuelle Plagnol-Diéval, «La presse contemporaine et l'œuvre romanesque de Mme de Genlis ", Journalisme et fiction au XVIII siècle, 20-23 septembre 1995, actes du colloque de l'Université d'Exeter, Malcolm Cook et Annie Jourdan (ed.), French Studies of the Eighteenth and Nineteenth Centuries, Peter Lang, Bern, Berlin, 1999, p. 197-212.

59. Mémoires, III, 171.

60. Ibid., III, 259.

61. Ibid., III, 173.

62. Ibid., III, 189.

63. Ibid., III, 190.

64. Ibid., IV, 12.

65. Ibid., IV, 22.

66. Ibid., IV, 40-43.

67. Ibid., IV, 43-45.

68. Ibid., III, 260.

69. Ibid., IV, 82. Avec cet argument final peu convaincant : « et j'ai osé dire : "Où peut-on mieux rêver à la gloire que dans les jardins de Chantilly" ?".

70. Ibid., III, 260.

71. Ibid., IV, 91. Les Mémoires de Louis-Philippe sont ainsi présentés par France Archives, portail national : Plan et ordre des matières des Mémoires jusqu'en avril 1793. Autographe. 5 cahiers de 4 pages.

TOME I. - Depuis sa naissance en 1773 jusqu'au 20 avril 1792, date de l'entrée en guerre de la France contre l'Autriche. Préambule sur ses intentions: relation fidèle des événements de sa vie et désir que ces mémoires soient publiés après sa mort. Éducation du prince par Mme de Genlis, États-généraux du 5 mai et autres événements de l'année 1789, appartenance au Club des Jacobins, séjours à Vendôme, Valenciennes, notes sur son père, l'émigration, etc.

Tome I, $1^{\text {re }}$ version. Copie avec corrections autographes, titres autographes: "Mes Mémoires commencés à Twickenham en $1802 » .1$ registre in- $4^{\circ}, 585$ pages.

Tome I, $2^{\mathrm{e}}$ version. Copie au net, Twickenham 1816. 1 registre in- $\mathrm{f}^{\circ}$, relié velours rouge, 279 pages. Exemplaire de luxe avec page de titre ornée de fioritures à la plume.

Tome I, $3^{\text {e }}$ version. Corrections autographes au crayon. 1 cahier in- $4^{\circ}, 585$ pages. Manuscrit autographe sur le duc d'Orléans, son père, fin 1791-janvier 1792. Cahiers 24 et 24bis, 6 pages. Note historique autographe sur la conduite du duc d'Orléans, son père, en 1787-1788. 4 pages in- $\mathrm{f}^{\circ}$. (Merlin et Freillard sont les deux députés ayant accompagné le Duc d'Orléans à la séance pour le vote sur la mort du roi Louis XVI) Notes historiques, anecdotiques, additions à faire, appréciations sur les notes, itinéraires, avec un état dressé par le général Dumas. 18 pièces.

TOME II. -Vie militaire du 20 avril au 30 septembre 1792. Guerre avec les AustroPrussiens, bataille de Valmy, entretien avec Danton.

Tome II, $1^{\text {re }}$ version [20 avril-8 octobre 1792]. $1^{\text {re }}$ copie par Depelchin avec corrections autographes. Par la suite, les événements de la fin de septembre et du début d'octobre 1792 ont été rapportés dans le tome III. 1 cahier in- $4^{\circ}, 389$ pages, quelques cahiers manquants. 
Tome II, $2^{\mathrm{e}}$ version, copie au net (20 avril-8 octobre 1792). 1 cahier in- $4^{\circ}, 396$ pages. Journal autographe des opérations et mouvements militaires, début juin-fin octobre 1792. 1 cahier in-12, pages 3 à 22 (p. 1 et 2 manquant). Journal autographe, affaires militaires, septembre 1791-20 juin 1792, 26 pages.

Tome II, $2^{\mathrm{e}}$ version. Relation autographe de Valmy, avec corrections autographes, 20 septembre 1792, 7 pages. Site : Archives de la maison de France (Branche Orléans). AP/ 300(III)/1-AP/300(III)/1052. Consultées le 17 mars 2018.

La BnF mentionne également le Mémorial des pensées et actions du duc de Chartres,... écrit par lui-même, en 1790 et 1791, tiré de la correspondance de Louis-Philippe-Joseph d'Orléans, avec Louis XVI, la reine, Montmorin, Liancourt, etc., imprimée et publiée en 1800 ; avec un discours préliminaire, notes et appendice, Paris, Delaunay fils, 1830. Mme de Genlis peut se référer à cette édition de 1800.

72. L'affirmation, déjà présente dans Les Leçons... est reprise dans les Mémoires, III, 143.

73. Ibid., IV, 304.

74. Ibid., IV, 315-316.

75. Ibid., III, 145.

76. Ibid., III, 174-176.

77. Ibid., III, 176.

78. Ibid., I, 23-24, 27, 37.

79. Ibid., I, 32.

\section{RÉSUMÉS}

Les Mémoires de Mme de Genlis, au-delà des sources autobiographiques et historiques, offrent un écart intéressant entre le contenu des œuvres éducatives qui jalonnent la carrière de l'auteure et le reflet qu'en donnent les volumes de 1825. En analysant comment les Mémoires... réécrivent la présentation, le contenu et la réception des ouvrages d'éducation, parus pour les premiers environ 40 ans avant, en dégageant les filtres interprétatifs mis en avant, on peut voir quelles fonctions complexes l'ancien "gouverneur » entend donner à ses souvenirs. C'est cette unité plausible, sinon factice, cette construction d'une continuité religieuse et morale, politique et sociale qui nous intéresse ici.

How to write a bio-bibliography: the retrospective look of Mme de Genlis on her educational books in her Mémoires. In the context of "Enlightenment", the Memoires of Mme de Genlis are interesting, not only as an autobiographical or historical source, but as a gap between the content of her different educational works and the reflection on this topic given by the volumes of 1825 . Our hypothesis is to show how the relationship to religion and politics in the pedagogical perspective changes and how Mrs de Genlis tries to build a posteriori a plausible unity when she talks about her educational works. 
INDEX

Keywords : Genlis, Memoirs, education, religion, morals, revolution

Mots-clés : Genlis, Mémoires, éducation, religion, morale, révolution

\section{AUTEUR}

MARIE-EMMANUELLE PLAGNOL-DIÉVAL

Université Paris Est Créteil, France 